\title{
An Ultrametric State Space with a Dense Discrete Overlap Distribution: Paperfolding Sequences
}

\author{
Aernout C.D. van Enter · Ellis de Groote
}

Received: 12 October 2010 / Accepted: 2 December 2010 / Published online: 21 December 2010

(C) The Author(s) 2010. This article is published with open access at Springerlink.com

\begin{abstract}
We compute the Parisi overlap distribution for paperfolding sequences. It turns out to be discrete, and to live on the dyadic rationals. Hence it is a pure point measure whose support (as a closed set) is the full interval $[-1,+1]$. The space of paperfolding sequences has an ultrametric structure. Our example provides an illustration of some properties which were suggested to occur for pure states in spin glass models.
\end{abstract}

\section{Introduction}

In [12], the study of the Parisi overlap distribution for various classes of non-periodically ordered sequences was undertaken.

The considered sequences were members of $\{-,+\}^{\mathbb{Z}}$ and their orbit closure forms typically a uniquely ergodic system, with a unique shift-invariant measure $\mu$. This measure then can be a ground state for a translation-invariant interaction which one can construct $[4,30,31]$, and the individual sequences will be the pure (extremal) ground states.

Their overlap distribution gives the behavior under the product measure of $\mu$ with itself, describing a two-replica system, written as $\mu \times \mu$ of the overlap between two randomly (from this product measure) chosen, bi-infinite sequences $\sigma$ and $\sigma^{\prime}$ :

$$
q_{\sigma \sigma^{\prime}}=\lim _{N \rightarrow \infty} \frac{1}{N} \sum_{i=1}^{N} \sigma_{i} \sigma_{i}^{\prime} .
$$

Note that the overlap $q_{\sigma \sigma^{\prime}}$ between two sequences is directly related to their Hamming distance $d_{H}\left(\sigma, \sigma^{\prime}\right)=\frac{1-q_{\sigma \sigma^{\prime}}}{2}$.

A.C.D. van Enter $(\bowtie)$

Johann Bernoulli Institute, Rijksuniversiteit Groningen, Nijenborgh 9, 9747 AG Groningen,

The Netherlands

e-mail: A.C.D.van.Enter@rug.nl

E. de Groote

De Vonderkampen 116, 9411RG, Beilen, The Netherlands

e-mail: ellisdegroote@ hotmail.com 
If we take two random sequences, each chosen according to the same shift-invariant measure, with probability one the above limit will exist, as follows from the ergodic theorem. The product measure of two ergodic measures, however, although shift-invariant, in general will not be ergodic, thus one may obtain different values for the overlap with positive probability.

A simple example illustrating this is the symmetric measure which give equal weights to the two alternating sequences:

$$
\mu_{a l t}(\sigma)=\frac{1}{2}(\delta(+-\ldots, \sigma)+\delta(-+\ldots, \sigma))
$$

This measure is ergodic under the shift transformation, but the product measure of $\mu_{\text {alt }}$ with itself is not. For the overlaps it holds that with probability $\frac{1}{2}$ the same sequence is chosen (overlap one) and with probability $\frac{1}{2}$ two different sequences are chosen (overlap minus one). Thus in this simple example

$$
p(q)=\frac{1}{2}(\delta(q, 1)+\delta(q,-1))
$$

In more general situations the overlap can take any value in the interval $[-1,+1]$, and the induced measure, which is the overlap distribution, describable by a probability distribution function on this interval, can be discrete ( that is, it is a pure point measure) or nondiscrete, and thus have continuous components.

In particular, it was found in [12] that whenever the atomic ("diffraction") spectrum of the unique translation-invariant measure on the sequences does not contain a pure point component, the overlap distribution is trivial (that is, it is concentrated at a single point); this applies in particular to all weakly mixing systems (in which case their product measures are ergodic), as well as to the (Prouhet-(Thue-))Morse system.

On the other hand for the Fibonacci sequences the overlap distribution was found to contain a(n absolutely) continuous part. In fact, in explicit form it is [12]

$$
p(q) d q=(2 \gamma-1) \delta(q, 1-4(1-\gamma))+\frac{1}{2} 1_{[1-4(1-\gamma), 1]}(q) d q
$$

where $\gamma=\frac{2}{1+\sqrt{5}}$.

We notice here that the same argument which was used in [12] applies to more general Sturmian sequences, which thus also have a continuous part in their overlap distribution. The reason is that these are also rotation sequences, see e.g. [19]. They also have recently been studied as ground states of some explicit interaction functions on lattice systems in [1]).

Moreover, it was found that the Toeplitz (or period-doubling) sequences give rise to an overlap distribution which is concentrated on a countable number of values, with 1 as its only limit point. Also, the set of these Toeplitz sequences has a tree (ultrametric) structure, and the overlap distribution could be obtained via this structure.

Here we show that the paperfolding sequences (see $[6,10]$ and references mentioned there), which also display such an ultrametric structure, give rise to an overlap distribution living on a dense countable number of points. As such the paperfolding system, which, in contrast to the Toeplitz system, is symmetric under the spin-flip (plus-minus) symmetry, is even closer to what is expected in Parisi's replica symmetry breaking theory of the Sherrington-Kirkpatrick model [20, 29]. In Parisi's theory for the SK model, this countable overlap distribution is supposed to show up for a fixed disorder, while averaging over 
the disorder distribution is supposed to result in a continuous piece in the overlap distribution. Often the countable overlap distribution is interpreted as being related to or implying a countable number of pure states; however there seems not to be much justification for this [9].

Conceptually, our example illustrates a number of points:

Newman and Stein [22-27] have shown that self-averaging arguments, based on the use of the ergodic theorem, apply to many finite-dimensional, disordered (spin-glass) models (although not to the equivalent-neighbor Sherrington-Kirkpatrick model). In particular they find that for systems with a spatial structure, in contrast to the Parisi theory's predictions for the Sherrington-Kirkpatrick model [20, 29], overlap quantities cannot depend on the realization of the disorder parameter, that is, they are "self-averaging". For example, for Edwards-Anderson-type spin-glass models there is no difference between a typical (Parisi SK-prediction: supported on countably many values, dense) and an averaged (Parisi SKprediction: continuous) overlap distribution.

As mentioned before, for many nonperiodic systems, the individual (nonperiodic) sequences are ground states for some translation invariant deterministic interaction. Thus they are often used as models for non-periodic (quasi-)crystals, [4, 14, 21, 30-33, 35]. It therefore becomes natural to consider various questions naturally arising in statistical mechanics about such systems, whether about their spectral properties [5, 13, 17, 18] or, as was done in $[11,12]$ and also here, about their overlap distributions. Typically, there are uncountably many such ground states, and as mentioned before, they can have nontrivial overlap distributions; somewhat surprisingly even without the presence of any disorder.

It turns out that:

- There is no general connection between the number of pure states -uncountable- and the number of possible overlap values - which can be one, countably or uncountably many.

- Triviality, non-triviality, and ultrametricity of the Parisi overlap distribution all can be realized without any role for the disorder.

- A dense discrete overlap distribution, with a hierarchical structure, can be realized (by the paperfolding system).

Our result illustrates the suggestion made in [16] that considering nonperiodic systems may be instructive for problems originating in the (spin-)glass community.

\section{Main Result}

For background on the paperfolding system we refer to $[2,3,6,10]$, and references mentioned there.

A paperfolding sequence can be constructed as follows:

In step 1 , we choose $k_{1}$ to be a number from the set $\{1,2,3,4\}$.

Now we fill all sites of the form $k_{1}+4 \mathbb{Z}$ with pluses, and all sites of the form $k_{1}+2+4 \mathbb{Z}$ with minuses.

Thus we have the period-4 structure covering half the sites:

$$
\ldots+.-.+.-.+.-.+.-\ldots
$$

In step 2, we again choose $k_{2}$ to be a number from the set $\{1,2,3,4\}$.

Now we occupy all sites of the form $k_{1}+1+2 k_{2}+8 \mathbb{Z}$ with pluses and all sites $k_{1}+5+$ $2 k_{2}+8 \mathbb{Z}$ with minuses. 
We repeat this step and in the $m$ th step we again occupy one quarter of the remaining empty sites with pluses, periodically with period $2 \times 2^{m}$, and the quarter of the sites at distance $2^{m}$ from the pluses we occupy with minuses, periodically with the same period. Thus in each step one half of the remaining sites are filled.

After making a choice for $k_{m}$, for all $m \in \mathbb{N}$, all sites (except possibly one) will be filled, and we have a paperfolding sequence (possibly with one site unspecified). Notice that in every step the uncountable set of sequences is divided into four times as many subsets as in the previous step, which generates the hierarchical (ultrametric) structure of the uncountable set of sequences.

With probability $\frac{1}{2}$ we find the overlap value $q=0$, which happens if in step 1 one "odd" and one "even" sequence was chosen, that is, $k_{1}-k_{1}^{\prime}$ is odd. With probability $\frac{1}{8}$ one finds overlap values $q$ equal to plus or minus $\frac{1}{2}$ respectively, which happens if in the first step either the same $\left(k_{1}=k_{1}^{\prime}\right)$, or the opposite $\left(\left|k_{1}-k_{1}^{\prime}\right|=2\right)$ choice was made, and in step 2 a different even-odd choice for $k_{2}$ and $k_{2}^{\prime}$. We repeat this argument, and we find that with probability $\frac{1}{2^{2 n+1}}$ one finds overlap values $\frac{(2 p+1)}{2^{n}}$, with $p=-2^{n-1}, \ldots, 2^{n-1}-1$ [15]. Thus in the end, the overlap distribution becomes concentrated on the dyadic rationals.

Summarizing we have proven:

Theorem 1 The overlap distribution of paperfolding sequences is given by:

$$
p(q)=\sum_{n=0}^{\infty} \sum_{m}^{\prime} \frac{1}{2}\left(\frac{1}{4}\right)^{n} \delta\left(q, \frac{m}{2^{n}}\right)
$$

where the primed sum indicates that the integers $n, m$ must satisfy the following conditions:

- if $n=0$ then $m=0$,

- if $n>0$ then $m$ is odd and $|m|<2^{n}$.

Comments Properties which are common between the period-doubling and the paperfolding system are:

(1) Limit periodicity. The sequences are a countable union of periodic subsequences of pluses and minuses. This implies a countable number of possible values for the overlap.

(2) There is a tree (hierarchical, ultrametric) structure in the set of sequences- the pure states. As the overlap between two sequences depends on the level of the tree at which a different even-odd choice is made, the overlap distribution reflects this.

(3) Both paperfolding and period-doubling sequences form model sets, obtained by a cut and project scheme, with an internal space of 2-adic numbers. [6-8, 34]. This agrees with the existence of a connection between Parisi's replica-symmetry-breaking ideas and p-adic numbers as was suggested in [28].

However, in contrast to the period-doubling case, the set of paperfolding sequences is spin-flip symmetric, and the set on which the overlap distribution is concentrated now lies dense in the interval $[-1,+1]$.

Acknowledgements A.C.D. v.E became interested in these issues during an earlier collaboration with Bert Hof and Jacek Miękisz. He also acknowledges very useful discussions with Michael Baake, Jacek Miękisz and Dimitri Petritis. We also thank Michael Baake for some helpful advice on the manuscript.

Open Access This article is distributed under the terms of the Creative Commons Attribution Noncommercial License which permits any noncommercial use, distribution, and reproduction in any medium, provided the original author(s) and source are credited. 


\section{References}

1. Anagnostopoulou, V., Díaz-Ordaz, K., Jenkinson, O., Richard, C.: Sturmian maximizing measures for the piecewise-linear cosine family. Dresden, preprint (2010)

2. Allouche, J.-P., Mendès France, M.: Automata and automatic sequences. In: Axel, F., Gratias, D. (eds.) Beyond Quasicrystals, pp. 293-367. Les Éditions de Physique/Springer, Berlin (1995)

3. Allouche, J.-P., Shallit, J.: Automatic sequences. Cambridge University Press, Cambridge (2003)

4. Aubry, S.: Weakly periodic structures and example. J. Phys. (Paris) C3-50, 97-106 (1989)

5. Baake, M., Höffe, M.: Diffraction of random tilings: Some rigorous results. J. Stat. Phys. 99, 219-261 (2000)

6. Baake, M., Moody, R.V., Richard, C., Sing, B.: Which distributions of matter diffract? Some answers. In: Trebin, H.-R. (ed.) Quasicrystals: Structure and Physical Properties, pp. 188-207. Wiley/VCH, New York/Weinheim (2003). arXiv:math-ph/0301019

7. Baake, M., Moody, R.V., Schlottmann, M.: Limit-(quasi)periodic point sets as quasicrystals with p-adic internal spaces. J. Phys. A, Math. Gen. 31, 5755-5765 (1998)

8. Baake, M., Moody, R.V.: Wighted Dirac combs with pure point diffraction. J. Reine Angew. Math. 573, 61-94 (2004). arXiv:math.MG/0203030

9. Bolthausen, E.: In: Bolthausen, E., Bovier, A. (eds.) Spin Glasses. Lecture Notes in Mathematics, vol. 1900. Springer, Berlin (2007). See in particular p. 16

10. Dekking, M., Mendes-France, M., van der Poorten, A.: FOLDS! (I,II and III). Math. Intell. 4, 130-138 (1982), p. 173-181, p. 190-195

11. van Enter, A.C.D.: On the set of pure states for some systems with non-periodic long-range order. Physica A 232, 600-607 (1996)

12. van Enter, A.C.D., Hof, A., Miękisz, J.: Overlap distributions for deterministic systems with many pure states. J. Phys. A, Math. Gen. 25, L1133-1137 (1992)

13. van Enter, A.C.D., Miękisz, J.: How should one define a (weak) crystal? J. Stat. Phys. 66, 1147-1153 (1992)

14. Gardner, C., Miękisz, J., Radin, C., van Enter, A.C.D.: Fractal symmetry in an Ising model. J. Phys. A, Math. Gen. 22, L1019-1023 (1989)

15. de Groote, E.: The overlap distribution of paperfolding sequences. Groningen bachelor thesis, 2010

16. Kurchan, J.: Dynamic Heterogeneities in Glasses, Colloids and Granular Matterials, Chap. 1. Oxford University Press, London (2011, to appear). arXiv:1010.2953. From Kurchan's answer to Question 10: "It is amazing how little attention the glass community has paid to lessons that could be learned from quasicrystals and in general nonperiodic systems."

17. Lee, J.-Y., Moody, R.V.: Lattice substitution systems and model sets. Discrete Comput. Geom. 25, 173$201(2001)$

18. Lee, J.-Y., Moody, R.V., Solomyak, B.: Pure point dynamical and diffraction spectra. Ann. Inst. Henri Poincaré, Theor. Math. Phys. 3, 1003-1018 (2002)

19. Lü, K., Wang, J.: Construction of sturmian sequences. J. Phys. A, Math. Gen. 38, 2891-2897 (2005)

20. Mézard, M., Parisi, G., Virasoro, M.A.: Spin Glass Theory and Beyond. Singapore, World Scientific (1987)

21. Miękisz, J.: Quasicrystals: Microscopic models of non-periodic structures. In: Leuven Lecture Notes in Mathematical and Theoretical Physics, vol. 5 (1993)

22. Newman, C.M.: Topics in Disordered Systems. ETH Lectures in Mathematics. Birkhäuser, Basel/Boston/Berlin (1997)

23. Newman, C.M., Stein, D.L.: The metastate approach to thermodynamic chaos. Phys. Rev. E 55, 5945211 (1997)

24. Newman, C.M., Stein, D.L.: Thermodynamic chaos and the structure of short-range spin glasses. In: Bovier, A., Picco, P. (eds.) Mathematical Aspects of Spin Glasses and Neural Networks, pp. 243-287. Birkhäuser, Boston/Basel/Berlin (1998)

25. Newman, C.M., Stein, D.L.: Ordering and broken symmetry in short-ranged spin glasses. J. Phys., Condens. Matter 15, R1319-R1364 (2003). arXiv:cond-mat/0301403, Topical Review

26. Newman, C.M., Stein, D.L.: The state(s) of replica symmetry breaking: Mean field theories versus shortranged spin glasses. (Formerly known as "Replica Symmetry Breaking's New Clothes"). J. Stat. Phys. 106, 213-244 (2002)

27. Newman, C.M., Stein, D.L.: Distribution of pure states in short-range spin glasses. Int. J. Mod. Phys. B24, 2091-2106 (2010)

28. Parisi, G., Sourlas, N.: P-adic numbers and replica symmetry breaking. Eur. J. Phys. B14, $235-242$ (2000)

29. Parisi, G., Talagrand, M.: On the distribution of the overlaps at given disorder. C. R. Acad. Sci., Ser. I Math. 339, 303-306 (2004) 
30. Radin, C.: Disordered ground states of classical lattice models. Rev. Math. Phys. 3, 125-135 (1991)

31. Radin, C.: Low temperature and the origin of crystalline symmetry. Int. J. Mod. Phys. B 1, 1157-1191 (1987)

32. Ruelle, D.: Do turbulent crystals exist? Physica 113A, 619-623 (1982)

33. Schechtman, D., Blech, I., Gratias, D., Cahn, J.W.: Metallic phase with long-range orientational order and no translation symmetry. Phys. Rev. Lett. 53, 1951-1953 (1984)

34. Schlottmann, M.: Generalized model sets and dynamical systems. In: Baake, M., Moody, R.V. (eds.) Directions in Mathematical Quasicrystals. CRM Monograph Series, vol. 13, pp. 143-159. AMS, Providence (2000)

35. Senechal, M.: Quasicrystals and Geometry. Cambridge University Press, Cambridge (1995) 